\title{
Exposing undergraduate students to research: A Condensed Matter Physics case study
}

\author{
Yuriko Baba ${ }^{1}$, Elena Díaz ${ }^{1}$, Francisco Domínguez-Adame ${ }^{1}$, Álvaro Díaz-Fernández ${ }^{2}$ \\ ${ }^{1}$ Departmento de Física de Materiales, Universidad Complutense de Madrid, Spain, \\ ${ }^{2}$ Departamento de Estructuras y Física de Edificación, Universidad Politécnica de Madrid, \\ Spain.
}

\begin{abstract}
In an effort to communicate major scientific breakthroughs to a vast audience, the media tends to present brief accounts of the findings and the process towards their discovery. While this may be sufficient for the layperson, it is certainly not enough for an undergraduate student in a scientific discipline. Moreover, the media will unlikely be able to judge the relevance of different breakthroughs and more emphasis will be put in those discoveries with more captivating titles. This is particularly worrying when it comes to actual students as it utterly biases their decisions when aiming to pursue a research career. The fact that syllabi tend to leave little to no room for introducing concepts that go beyond the standard curriculum leaves this problem unsolved. With the aim of tackling these issues, we have organized a workshop where experts from various institutions delivered lectures and even performed exhibitions of the phenomena being discussed. The initiative, named Recent Advances in Condensed Matter Physics, was aimed at undergraduate Physics students in their last two years at our University. In order to assess the strategies of our learning methodology, the experiment was carried out for three consecutive academic years and feedback from students was collected in the form of homework and surveys. The results show that introducing recent research discoveries in the curricula is a complex yet profitable strategy.
\end{abstract}

Keywords: Learning methodologies; undergraduate initiation in research; student guidance; condensed matter physics. 


\section{Introduction}

The term Condensed Matter Physics (CMP) was coined by Philip Warren Anderson (Nobel Prize in Physics in 1977) and Volker Heine in 1967, when they were working at the Cavendish Laboratory, Cambridge. This subject deals with condensed phases of matter, namely, solids and liquids with many constituents (electrons and ions) under strong interactions between them and possibly subjected to external electromagnetic fields. According to the number of published papers, CMP is the most active sub-field of research in Physics nowadays (Sinatra 2015). Since the year 2000, the Royal Swedish Academy of Sciences has awarded the Nobel Prize in Physics six times to researchers working in areas related to CMP, including the last one in 2016. Besides the interest in fundamental aspects of Physics, CMP is a key area for the development of new technologies in a variety of niches (quantum and information technologies, data storage, energy harvesting and tailored materials for an endless series of applications, to name a few of them). A large number of scientists trained in CMP work in industry and found the training they received in University very rewarding.

The vast majority of undergraduate Physics degrees, if not all of them, offer subjects related to CMP, from introductory levels (mostly referred to as Solid State Physics courses) to advanced ones. However, these subjects' syllabi are often exceedingly dense and broad, with a large range of topics to be covered. As a result, there is usually very little time to present recent scientific discoveries. Such discoveries are often briefly accounted for in the media and sometimes may even go unnoticed for their not being sufficiently captivating. This asynchrony between the content delivered in the standard curriculum and the discoveries at the forefront of science leaves a considerable gap that students cannot overcome until attending graduate courses. By that time, students have already chosen a path to follow in their research without having had enough information about novel findings in different fields of the physical sciences.

Throughout the years, we have been able to observe how students perceive the aforementioned asynchrony between the concepts being taught in their lectures and the current research in fields such as CMP. A great example is that of graphene, a material which, since its experimental discovery in 2010, has led to a plethora of revolutionary discoveries. In contrast, students in their undergraduate courses will learn that graphene is a crystalline material with interesting properties but not much more. An excellent review on how to bridge the gap between teaching and research-based teaching can be found in Fraser et al. (2014). With this in mind, we decided to put the idea of drawing students closer to recent advances in CMP to the test, by bringing together their knowledge from their standard lectures and the most recent discoveries, as well as explaining how these have reshaped our modern world. In this work, we will present the initiatives and activities that were carried out during the academic years 2017/18, 2018/19 and 2019/20, along with the main results and conclusions. 


\section{Methodology and work plan}

\subsection{Objectives}

Our work revolves around the workshop Recent Advances in Condensed Matter Physics (RACMP). All other activities and the data collection stem from the workshop. More specifically, our goals when designing this project have been:

- To make students familiar with the most recent and leading research topics in the area of CMP.

- To increase the interest in pursuing a research career and, in particular, to draw the students' attention to CMP.

- To statistically assess the students' response by means of surveys. In particular, we intended to find whether their being exposed to advances in CMP increased their awareness on the importance of CMP-related subjects within their degree.

- To show the results and conclusions to the academic authorities of our Department with the objective of introducing new methodologies inside the classroom.

\subsection{Expected impact and indicators}

The expected impact is very specific and easily quantifiable. On the one hand, there is a series of activities which will be discussed in detail later in the paper. Thus, the organization of the workshop, the seminars, the number of participants, the number of optional European Credit Transfer and Accumulation System (ECTS) credits, the speakers, etc. already provide indicators related to the statistical samples that have been used for the surveys. On the other hand, we have the actual surveys, the answers to which are found in Section 3. Finally, a White Book with specific proposals shown to the academic authorities in our Department is included in Section 4. We expect the impact to be immediate since the updates in the syllabi and the new methodologies are readily accessible for their introduction in the current curriculum.

\subsection{Organization}

The workshop RACMP took place during the second semester of each academic course, from February to May, during the academic courses 2017/18, 2018/19 and 2019/20. The students in mind were third- and fourth-year undergraduate students in Physics. Each session was 90 minutes long and they took place once a week during 10 weeks. Finding the right timing for the lectures was not straightforward since students in their last courses have a broad range of optional subjects, which massively overlap. Each session was delivered by experts in CMP, both from academia, research laboratories and scientific institutions (see Figure 1). The invited researchers were delighted with the idea and promptly accepted the proposal. The number of students who attended the workshop each academic year was above 60, much 
higher than those attending the optional subjects in CMP. Most students attended the seminars regularly and received a certificate of participation and attendance. Students were also given the possibility to hand in a summary no longer than 1000 words related to one of the sessions of their own choosing. Those who did could apply for 1 optional ECTS credit.

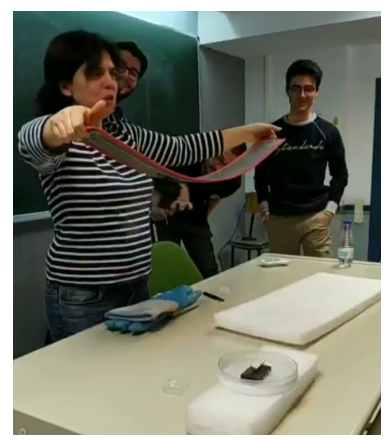

Figure 1. Prof. María José Calderón, from Instituto de Ciencia de Materiales de Madrid (Consejo Superior de Investigaciones Cientificas), in a classroom demonstration of the amazing physics of high-temperature superconductors.

\subsection{Topics}

The titles of the sessions in the academic year 2017/18 were the following

(1) Electronic correlation: From jellium to supercomputation.

(2) The quantum Hall effects.

(3) Topology at the service of condensed matter.

(4) Dirac materials based on graphene: CERN in miniature.

(5) Quantum dots: Manipulation and control of artificial atoms.

(6) Chiral molecules: Spintronics without magnets.

(7) Magnetic nanowires: A route towards new sensors.

(8) From Foucault's pendulum to Hofstadter's butterfly: creating synthetic gauges in condensed matter.

(9) Magnetic tunnel effect: Versatility of complex oxides.

(10) Exotic fluids and granular matter: From fundamental physics to silos.

The titles of the sessions in the academic year 2018/19 were the following

(1) The quantum Hall effects.

(2) Irreversibility and dissipation in microscopic engines.

(3) Chiral molecules: Spintronics without magnets.

(4) Superconductivity.

(5) Optical properties of semiconducting nanowires.

(6) Graphene nanostructures. 
(7) Emergent phenomena: interfaces.

(8) Mechanical properties and friction at the nanoscale.

(9) Correlated oxides interfaces' based spintronics.

(10) Thermal transport at the nanoscale.

The titles of the sessions in the academic year 2019/20 were the following

(1) Chiral molecules: Spintronics without magnets.

(2) Topological insulators and topological semimetals.

(3) The quantum Hall effects.

(4) Twisted bilayer graphene: the magic of Moiré.

(5) Optical properties of semiconducting nanowires.

(6) Quantum dots: a versatile platform towards quantum computation and quantum simulation.

(7) Strengths and weaknesses of ab-initio calculations.

(8) Nanowires: a route towards efficient thermoelectric devices.

(9) Superconductivity.

(10) Thermal transport at the nanoscale.

Due to the Covid-19 crisis, the last five sessions of the course 2019/20 were delivered online.

\section{Survey}

\subsection{Survey questions}

At the end of the workshop, students were given anonymous surveys which contained the following questions:

(1) Have you taken or are currently taking Condensed Matter Physics in your undergraduate or graduate studies?

(2) Did you know what Condensed Matter Physics was before attending the workshop?

(3) After this workshop, has your interest in deepening your knowledge through other courses, activities or optional subjects on topics related to Condensed Matter Physics increased?

(4) Would you like to pursue a career in Condensed Matter Physics?

(5) If so, which aspects are you most interested in? Theoretical, experimental or both.

(6) Do you think that the number of subjects offered in Condensed Matter Physics is proportional to its relevance?

(7) Do you think that the workshop format, with specific talks on current research topics, is interesting and applicable to other subjects of your degree?

(8) Are you considering applying for the optional credit?

(9) If so, do you think the evaluation method to be appropriate? 
(10) Would you attend the workshop again next year if new topics are introduced?

(11) Would you recommend your classmates the assistance to the workshop?

(12) Which topics did you miss in this year's workshop?

Data for the statistics: Male Female Other

\subsection{Results}

Table 1 presents a summary of the data retrieved from the survey.

Table 1. Statistics of answers to survey questions.

\begin{tabular}{cccc}
\hline Question & Yes & No & DK/NA \\
\hline 1 & $18,5 \%$ & $81,5 \%$ & 0 \\
2 & $70,4 \%$ & $29,6 \%$ & 0 \\
3 & $88,9 \%$ & $11,1 \%$ & 0 \\
4 & $48,2 \%$ & $40,8 \%$ & $11,0 \%$ \\
6 & $40,7 \%$ & $59,3 \%$ & 0 \\
7 & $92,6 \%$ & $7,4 \%$ & 0 \\
8 & $18,5 \%$ & $81,6 \%$ & 0 \\
9 & $100,0 \%$ & 0 & 0 \\
10 & $92,6 \%$ & $7,4 \%$ & 0 \\
11 & $96,3 \%$ & 0 & $3,7 \%$ \\
\hline
\end{tabular}

Question 5 was a follow-up question to question 4 which, in case of the latter being affirmative, asked about the student's interest in theoretical or experimental aspects in CMP. The responses were the following

Table 2. Question 5.

\begin{tabular}{ccccc}
\hline Question & Theory & Experiment & Both & DK/NA \\
\hline 5 & $14,8 \%$ & $33,3 \%$ & $29,6 \%$ & $22.3 \%$
\end{tabular}

In response to question 12, students missed talks on plasmonics, social repercussions of CMP and their applications, as well as visits to research laboratories. 


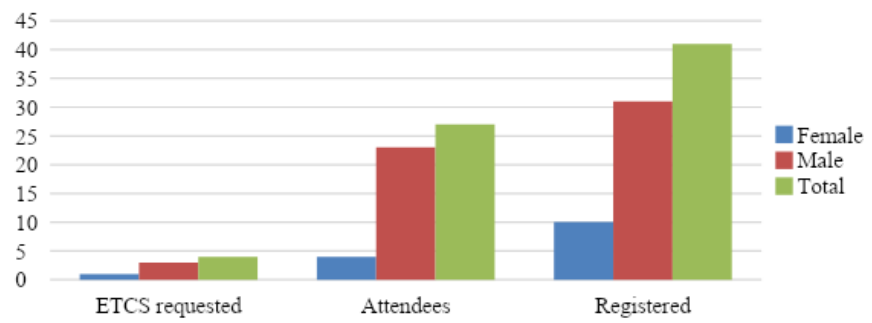

Figure 2. Gender of registered, attendees and ECTS requested to RACMP surveyed in 2018/19.

The data used for Figure 2 corresponds to the academic year 2018/19.

\subsection{Analysis of results}

From the results obtained in questions 1 and 2, regarding their knowing of CMP and their having taken courses on CMP before the seminars, most students replied that they did know of the existence of CMP although had not yet been enrolled in any subject related to it. This is very likely due to the fact that most students know that the syllabus of Solid State Physics encompasses topics that belong to the broader area of CMP. Question 3 shows that the workshop has fulfilled our objective of raising the student's interest on CMP since most students feel an urge to continue deepening their knowledge on the topics. Moreover, half of the attendees consider the possibility of pursuing a career in CMP (question 4), in a proportion that is even higher than the proportion of Physicists actually working in CMP. The interest in purely theoretical aspects is low, around a $15 \%$ (question 5) and, rather remarkably, all interested participants were male. In contrast, all participants who marked an interest in purely experimental aspects were female. It is interesting however to point out that about a third of the participants would like to pursue a mixed career, neither purely theoretical nor purely experimental. As question 6 shows, a large fraction of students, around $60 \%$, considers that CMP is underrepresented in the current curriculum of the Department of Physics. The responses to question 7 show that most students believe this kind of activity to be applicable to other subjects. Although not a large number of students were willing to apply for the optional credit (question 8), all who did consider the evaluation method to be appropriate (question 9). Finally, we would like to stress the large number of students (above $90 \%$ ) who would attend the following year's workshop and recommend it to their fellow classmates (questions 10 and 11).

In the optional comments, the attendees stress their interest in the proposed activity since it bring the researchers and their work closer to the students. Moreover, the low number of ECTS requests, even if the students consider the evaluation method to be appropriate (question 9), exhibits the genuine interest generated among the students by the CMP. 
Regarding the gender of the students, the data reflects the gender participation in our University, with a proportion of $36 \%$ female (Nieto-Zayas 2013). On the other hand, the speakers where exactly half of them male and half female, in mean value spanning the three years of activity.

\section{White book}

From the results obtained in the surveys as well as from the experience gained by the organizers, we made the following recommendations to our Department:

- In the first subjects of the Degree in Physics where students start being exposed to the behaviour of solid matter, namely, Materials Physics and Solid State Physics, it is convenient to emphasize that CMP represents a third of the current research in modern Physics.

- Since true modifications of the syllabi can be rather complex, alternatives must be put forward so as to show students the relevance of CMP. The RACMP workshop proved to be an excellent platform to achieve this aim, although it is clearly not the only alternative that exists.

- Offering optional credits favours the participation, even if the student already had a motivation to attend the activity.

- The workshop has allowed for the analysis, synthesis and evaluation of the contents on CMP from the students' viewpoint.

\section{Conclusions}

The surveys show that the RACMP constituted an interesting activity for students to get to know research at the forefront of Physics and to increase their interest in the subject. The results were included in a white book with specific purposes which were then shown to the authorities in our Department. The expected result is that improvements will be included in the teaching methodologies as well as in the syllabi. Finally, taking into account the students' interest and the need for an enhancement of the role of CMP in the standard curriculum, a fact that is not exclusive to our University, we believe that the results presented herein will be of interest to other Universities.

\section{Acknowledgments}

The authors thank Dunkan Martínez for helpful discussions. This work has been supported by Ministerio de Ciencia e Innovación (Grant PID2019-106820RB-C21). 


\section{References}

Fraser, J. M., Timan, A. N., Miller, K., Dowd, J. E., Tucker, L., \& Mazur, E. (2014). Teaching and physics education research: bridging the gap. Reports on Progress in Physics, 77(3), 032401. doi: 10.1088/0034-4885/77/3/032401.

Sinatra, R., Deville, P., Szell, M., Wang, D., \& Barábasi, A. L. (2015). A century of physics. Nature Physics, 11, 791-795. doi: 10.1038/nphys3494.

Nieto-Zayas, C. et al. (2013). Estudio sobre la inserción laboral de los licenciados en Física Promociones de los años 2009, 2010 y 2011. Universidad Complutense de Madrid, Serie informes 01/2013: Estudio sobre la inserción laboral de los licenciados en Física. https://www.ucm.es/data/cont/docs/3-2014-12-04-F\%C3\%8DSICA.\%202013.pdf 